\title{
Effect of transport of growing nanoparticles on capacitively coupled rf discharge dynamics
}

\author{
I. V. Schweigert, ${ }^{1,2}$ A. L. Alexandrov, ${ }^{1}$ D. A. Ariskin,,${ }^{1,2}$ F. M. Peeters, ${ }^{2}$ I. Stefanović, ${ }^{3,4}$ E. Kovačević, ${ }^{3}$ \\ J. Berndt, ${ }^{3}$ and J. Winter ${ }^{3}$ \\ ${ }^{1}$ Institute of Theoretical and Applied Mechanics, Russian Academy of Sciences, Novosibirsk 630090, Russia \\ ${ }^{2}$ Departement Fysica, Universiteit Antwerpen, Groenenborgerlaan 171, B-2020 Antwerpen, Belgium \\ ${ }^{3}$ Institute for Experimental Physics II, Ruhr-University Bochum, 44780 Bochum, Germany \\ ${ }^{4}$ Institute of Physics, Belgrade University, P.O. Box 57, 11000 Belgrade, Serbia
}

(Received 7 December 2007; revised manuscript received 20 June 2008; published 19 August 2008)

\begin{abstract}
We present experimental and numerical studies of the properties of a capacitively coupled $13.56 \mathrm{MHz}$ discharge in a mixture of $\mathrm{Ar}$ and $\mathrm{C}_{2} \mathrm{H}_{2}$ with growing nanosize particles. It is found that at the initial stage of the growth, nanoparticles are accumulated near the sheath-plasma boundaries, where the ionization by electrons is maximal. The nanoparticles suppress the ionization due to the absorbing fast electrons and stimulate a quick change of the plasma parameters followed by a transition between different modes of discharge operation. At that moment the peaked distribution of the dust particles transforms into a flat one.
\end{abstract}

DOI: 10.1103/PhysRevE.78.026410

PACS number(s): 52.27.Lw, 52.25.-b, 52.65.-y, 52.80.Pi

\section{INTRODUCTION}

Capacitively coupled $13.56 \mathrm{MHz}$ discharge in a mixture of argon and acetylene was intensively studied in recent experiments $[1,2]$. In the discharge volume due to gas phase reactions, the formation and growth of hydrocarbon nanoparticles were observed. The nanoparicles grow to some critical radius, leave the central part of the discharge, and eventually drop to the bottom electrode due to gravity. After that, a new particle growth cycle starts. This periodic behavior is accompanied by strong periodic variations of all plasma characteristics $[1,2]$. One of the most spectacular experimental observation is that only at the initial stage of nanoparticle growth do the discharge parameters change very rapidly. With further particle growth, only slight variation of the plasma parameters was found.

In this work, we concentrate our theoretical and experimental study on the phenomena of dust particle transport and the change of plasma parameters at the initial stage of particle growth. Note that the influence of movable dust on the discharge properties and the influence of the dust distribution on the initial stage of the nanoparticle growth are not completely understood. Previously, a numerical and experimental study of the capacitively coupled radiofrequency (CCRF) discharge with $50 \mathrm{~nm}$ unmovable dust particles in argon was carried out in Ref. [3]. The transition between capacitive and resistive modes of CCRF discharge operation was observed in pure silane with silicon particles in the experiment [4] and in numerical calculations [5]. In Ref. [6], the power absorption and discharge glow were analyzed within the framework of an analytical discharge model with uniformly distributed dust for the experimental conditions of Refs. [1,2]. The dust transport in the fluid approximation was studied in Ref. [7] for a two-dimensional geometry of the discharge chamber. The charging of nanoparticles was treated with using the OML theory and a Maxwellian velocity distribution of electrons and ions. However, in the dusty plasma it generally is not a good approximation, because electron and ion distribution can be far from Maxwellian. In Ref. [8], the charging of unmovable dust was studied in particle-in-cell Monte Carlo
Collision (PIC-MCC) simulations. The authors assumed that the nanoparticles were uniformly distributed over discharge volume including the electrode sheaths. The assumption is very rough, diminishing the reliability of the conclusions. The characteristic time of dust motion is much larger than the discharge rf time, and the nanoparticles move in a timeaveraged electrical field. Therefore, the negatively charged dust is trapped in the bulk plasma by the electrical potential.

New aspects in our study of CCRF discharge plasma with nanoparticles are as follows: (a) we consider dust particle charging by using the electron and ion energy distribution functions taken from the kinetic PIC-MCC simulation, (b) the motion of dust is calculated self-consistently with the discharge parameters, and (c) we analyze the role of movable dust on the transition process between the different discharge regimes.

The paper is organized as follows. The physical model of discharge plasma with movable dust is presented in Sec. II. The description of the numerical algorithm is given in Sec. III. In Sec. IV, we discuss the calculation of the ion drag force. The computed and measured plasma parameters and dust profiles for different nanoparticle sizes are presented in Sec. V. Our results are summarized in Sec. VI.

\section{PHYSICAL MODEL OF DISCHARGE PLASMA WITH MOVABLE DUST}

The physical model of the gas discharge plasma with charged massive dust particles is based on the kinetic description of the electron and ion motion and their interaction with charged nanoparticles. The distribution functions for electrons $f_{e}(t, x, \vec{v})$ and for ions $f_{i}(t, x, \vec{v})$ are calculated from the Boltzmann equations

$$
\begin{gathered}
\frac{\partial f_{e}}{\partial t}+\vec{v}_{e} \frac{\partial f_{e}}{\partial x}-\frac{e \vec{E}}{m_{e}} \frac{\partial f_{e}}{\partial \vec{v}_{e}}=J_{e}, \quad n_{e}=\int f_{e} d \vec{v}_{e}, \\
\frac{\partial f_{i}}{\partial t}+\vec{v}_{i} \frac{\partial f_{i}}{\partial x}+\frac{e \vec{E}}{m_{i}} \frac{\partial f_{i}}{\partial \vec{v}_{i}}=J_{i}, \quad n_{i}=\int f_{i} d \vec{v}_{i},
\end{gathered}
$$

where $v_{e}, v_{i}, n_{e}, n_{i}, m_{e}$, and $m_{i}$ are the velocities, densities, and masses of electrons and ions, respectively, $E$ is the elec- 
trical field, and $J_{e}, J_{i}$ are the collisional integrals for electrons and ions, which include elastic and inelastic scatterings with Ar atoms and $\mathrm{C}_{2} \mathrm{H}_{2}$ molecules, as well as scattering on charged nanoparticles. The absorption cross sections of the nanoparticle surface for electrons with energy $\epsilon_{e}>\phi_{d}$ is $\sigma_{e d}=\pi r_{d}^{2}\left(1-\phi_{d} / \epsilon_{e}\right)$ and for ions is $\sigma_{i d}=\pi r_{d}^{2}\left(1+\phi_{d} / \epsilon_{i}\right)$, where $r_{d}, \phi_{d}$ are the dust particle radius and surface potential, and $\epsilon_{e}, \epsilon_{i}$ are the electron and ion energies, respectively.

The charge of the dust particles $Z(x)$ as a function of the coordinate $x$ is calculated from the balance equation of the electron and ion currents on the dust surface,

$$
j_{e d}(x)=j_{i d}(x) .
$$

The density of charged nanoparticles $n_{d}$ is described with the continuity equation in the drift-diffusion approximation,

$$
\frac{\partial n_{d}}{\partial t}=\frac{\partial}{\partial x}\left(\frac{\partial D_{d} n_{d}}{\partial x}-\mu_{d} E Z n_{d}\right),
$$

where $D_{d}$ and $\mu_{d}$ are the diffusion and mobility coefficients, which include the electrostatic and ion drag forces acting on the charged nanoparticles. Here the internal pressure in the particle system can be neglected. For our system of nanoparticles, the coupling parameter $\Gamma=V /|r| k_{B} T$ (where $V$ is the electrostatic energy of the particle interaction and $k_{B} T$ is the particle kinetic energy) varies from 1 to 10 . This value is far below the critical coupling parameter for crystallization, which is 170 for a 3D Coulomb system. The screening of the nanoparticles in the ion background lowers the Coulomb interaction energy

$$
V(r)=\frac{Z^{2}}{\epsilon|r|} \exp \left(-|r| / \lambda_{i}\right),
$$

where $\epsilon$ is the dielectric constant, $r$ is the interparticle distance, and $\lambda_{i}$ is the ion screening width.

The electrical potential distribution $\phi$ obeys the Poisson equation

$$
\Delta \phi=4 \pi e\left(n_{e}-n_{i}+Z n_{d}\right), \quad E=-\frac{\partial \phi}{\partial x} .
$$

The boundary conditions for the Poisson equation are $U(d, t)=U_{0} \sin (\omega t)$ and $U(0, t)=0$, where $U_{0}$ is the applied voltage amplitude, and $x=0, x=d$ are the coordinates of electrodes.

As follows from the measured mass spectra in Ref. [1] several types of ions are generated in the mixture of Ar and $\mathrm{C}_{2} \mathrm{H}_{2}$ and the majority of ions are $\mathrm{C}_{n} \mathrm{H}_{2}$. To simplify the discharge model, we consider only one type of ion, $\mathrm{C}_{4} \mathrm{H}_{2}{ }^{+}$. Only elastic scattering of $\mathrm{C}_{4} \mathrm{H}_{2}{ }^{+}$ions in an Ar background is considered as the mixture consists of $94.2 \% \mathrm{Ar}$ and $5.8 \%$ $\mathrm{C}_{2} \mathrm{H}_{2}$. The electron scattering cross sections were taken from Refs. $[9,10]$ for argon and from Ref. [11] for acetylene.

\section{ALGORITHM OF CALCULATION}

The computational algorithm can be separated into the following main steps:

(a) The calculation starts from some initial distributions for the electron and ions taken from the analytical approxi- mation of discharge plasma. For dust particles density,

$$
\begin{gathered}
n_{d}(x)=N_{d} \sin (\pi x / d), \quad l_{s}<x<d-l_{s}, \\
n_{d}(x)=0, \quad x<l_{s}, \quad x>d-l_{s},
\end{gathered}
$$

where $l_{s}=1.5 \mathrm{~cm}$ is the initial width of the electrode sheath and $N_{d}=10^{7} \mathrm{~cm}^{-3}$. The initial nanoparticle floating potential is $3 \mathrm{~V}$. The conditions of quasineutrality of bulk plasma at $l_{s}<x<d-l_{s}$ are fulfilled and $n_{e}-n_{i}+Z n_{d}=0$.

(b) The electrical field distribution is calculated from the Poisson equation (5). We take into account the space charge of dust particles, electrons, and ions.

(c) The electron energy distribution function (EEDF), the ion energy distribution function (IEDF), and all macroscopic discharge parameters are calculated. The kinetic equations of motion for electrons and ions (1) and (2) are integrated in the electrical field of discharge taking into account collisional events with the background gas and dust particles. The probabilities of collisional events (elastic scattering, excitation of molecules, ionization, absorption on the dust surface, etc.) are set by the cross sections depending on the electron or ion energy and calculated with the Monte-Carlo technique and the null-collision method [12]. The cross section of electron or ion absorption is proportional to the dust density.

(d) As a next step, we calculate the dust floating potential. The PIC-MCC method gives the opportunity for a direct calculation of the electron and ion currents on the dust surface. During the Monte Carlo calculation of the electron and ion trajectories, we simply integrate the absorption events for electrons and ions on the dust particle surfaces. The electron and ion currents on the dust surface are averaged over the last 10-50 rf cycles (depending on the dust radius) in order to reduce the statistical fluctuations. After every rf cycle, we adjust the floating potential to improve the electron and ion current balance. This change is always less than $10 \%$ from the previous value to avoid any numerical instability due to a local jump of the electrical field.

(e) The electrostatic and ion drag forces acting on the dust particles are calculated at every rf cycle using the electrical field, ion density, and ion temperature averaged over the last $100 \mathrm{rf}$ cycles, as described in next section.

(f) The dust density distribution is found from the solution of the continuity equation (4). The interaction of dust particles is calculated through the iterative self-consistent solution of the kinetic equations (1) and (2), the continuity equation (4) and the Poisson equation (5). The dust particles move in the electrical field created by the distribution of the dust particles, electrons, and ions.

Then we return to (b) and calculate the electrical field from the Poisson equation with the new dust charge, electron, and ion distributions. The iterative procedure is continued until the steady-state solution is reached. Typically it takes about $1000 \mathrm{rf}$ cycles for discharge without dust and $5000 \mathrm{rf}$ cycles with dust to reach convergence. Note that in every run, the nanoparticle size is constant. The discharge can reach the steady state with the constant nanoparticle radius, because the characteristic time of plasma relaxation depending on electron and ion motion is much less than the characteristic time of dust growth. The number of artificial 
particles to mimic the electrons and ions in our PIC-MCC simulations is $N_{e}=N_{i}=10^{4}-10^{5}$ depending on the discharge parameters. The time step is $10^{-11} \mathrm{~s}$ for electrons and 3 $\times 10^{-10} \mathrm{~s}$ for ions. The interelectrode distance is divided by a grid with 201 nodes for the definition of the macroscopic plasma parameters and the calculation of the dust density distribution.

\section{ION DRAG FORCE}

For the definition of the ion drag force $F_{\mathrm{dr}}$, first we applied the method described in Ref. [13]. There the model system consists of charged particles in the ion flux in a constant external electrical field. The ion motion around the negatively charges dust particles is described with the (2D3V) kinetic Eq. (1) in cylindrical coordinates. The scatterings of ions (resonant charge exchange and elastic collisions) are taken into account. The electrons obey a Boltzmann distribution. The electric potential perturbation around the dust particle is found from the linearized Poisson equation in cylindrical coordinates. We have calculated the ion drag force for a set of plasma parameters using the density of the particle $n_{d}=10^{6}-10^{7} \mathrm{~cm}^{-3}$. In the discharge the negatively charged nanoparticles are screened by ions. The interparticle distance is $60-120 \mu \mathrm{m}$. The ion Debye screening length $\lambda_{D}$ is $30-60 \mu \mathrm{m}$ for $n_{i}=\left(2 \times 10^{9}\right)-\left(5 \times 10^{8}\right) \mathrm{cm}^{-3}$, and $T_{i}=0.03-0.5 \mathrm{eV}$. These results are not discussed in this paper because for our experimental conditions the ion drag forces calculated using the PIC-MCC method [13] and with the Barnes formula [14] coincide within 10\% accuracy, which is the statistical error of our Monte Carlo calculations. The Barnes formula describes accurately the ion drag force for nanoscale dust. Therefore, for the calculation of dust motion, we use the orbit part of the ion drag force that refers to the Coulomb interaction of ions with a nanoparticle $F_{\mathrm{dr}}(x)$ $=4 \pi m_{i} n_{i} v_{i} v_{s} b_{p}^{2} L$, where $b_{p}=Z e^{2} / 2 \epsilon_{i}$ is the effective interaction radius, $v_{i}$ is the ion drift velocity, $v_{s}=\left(2 \epsilon_{i} / m_{i}\right)^{1 / 2}$ is the mean ion velocity, $L=0.5 \log \left[\left(\lambda_{D}^{2}+b_{p}^{2}\right) /\left(b_{p}^{2}+b_{c}^{2}\right)\right]$ is the Coulomb integral, and $b_{c}^{2}=r_{d}^{2}\left(1+\phi_{d} / \epsilon_{i}\right)$ is the collection radius. The contribution to the ion drag force from captured ions is small for $r_{d}<100 \mathrm{~nm}$.

\section{TRANSITION BETWEEN DIFFERENT MODES}

Our simulations and measurements were performed for the $13.56 \mathrm{MHz}$ discharge operating in a mixture of $\mathrm{C}_{2} \mathrm{H}_{2} / \mathrm{Ar}$ (1:16) at $P=70$ mTorr. The discharge chamber consists of a parallel plate electrode system with $7 \mathrm{~cm}$ interelectrode distance. The detailed description of our experimental setup and measuring technique can be found elsewhere $[1,2]$. In the simulations, we assumed the voltage waveform to be sinusoidal, $U(t)=U_{0} \sin (\omega t)$, where $U_{0}$ is the voltage amplitude. The growth of nanoparticles from gas phase reactions is not considered in calculations. We took values of the voltage amplitude and dust radius from the experiment. In Fig. 1 the measured voltage amplitude and nanoparticle radius for $t$ $>1.5 \mathrm{~min}$ are given as a function of time. At first the discharge glows in pure argon, and at $t=0$ acetylene is added and the particle formation starts. Since there are no data

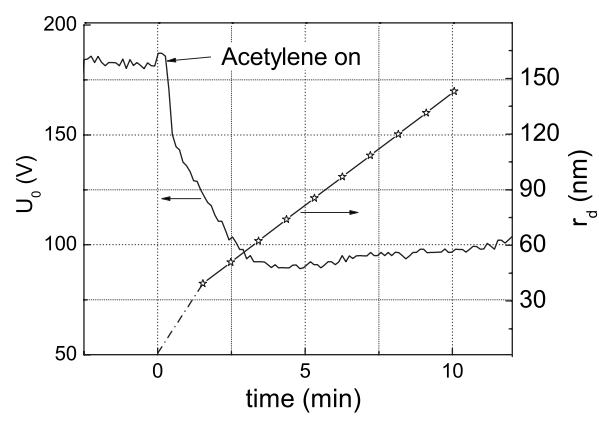

FIG. 1. Measured voltage amplitude (solid line) and measured dust radius (symbols) as a function of time.

about the dust radius on a very early stage of the growth, the dust radius is assumed to be growing linearly at $t<1.5 \mathrm{~min}$.

In our simulation as well as in the experiment, the dust has a monodisperse size. In our calculations, the nanoparticle radius varies from 10 to $90 \mathrm{~nm}$.

Without nanoparticles, the argon discharge glows in the capacitive mode, which is characterized with the large plasma density and low electron energy in the bulk plasma. Acetylene adding initiates the particle formation and the change of plasma parameters shown in Figs. 2 and 3. The dust acts as an electron and ion sink and therefore the plasma parameters are sensitive to an increase of nanoparticle surface area. However, only during the first 1.7 minutes does the electron density $n_{e}$ shown in Fig. 2 rapidly decrease. With further dust growth, $n_{e}$ remains constant up to the end of the dust growth cycle.

Figure 3 shows the evolution of the mean electron energy $\epsilon_{e}$ and ionization rate profiles. For smaller dust radii, $r_{d}$ $<20 \mathrm{~nm}$, the mean electron energy has maxima near the sheath-plasma boundaries. For larger radii, $r_{d}=30$ and $40 \mathrm{~nm}$, the $\epsilon_{e}$ profile changes qualitatively, denoting the transition from the capacitive (C) to volume-dominated (VD) mode. The ionization rate distribution also demonstrates this transition [Fig. 3(b)]. In the argon discharge, the ionization preferably takes place near the sheath-plasma boundary and it is very low in the bulk plasma. There the electrons are trapped by the electrical potential, thermalized, and have a Maxwellian energy distribution. Dust growing to $20 \mathrm{~nm}$ suppresses the ionization, but the discharge still glows in the capacitive regime. For 30 and $40 \mathrm{~nm}$ dust, the new VD regime is associated with an increase of the ionization rate in

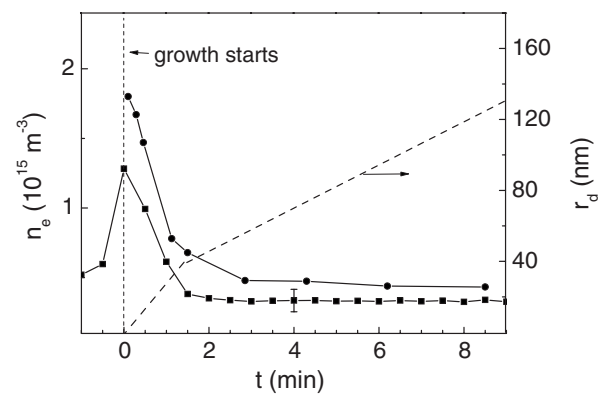

FIG. 2. Measured (squares) and calculated (circles) electron density in the midplane and measured nanoparticle radius (dashed line) as a function of time. 


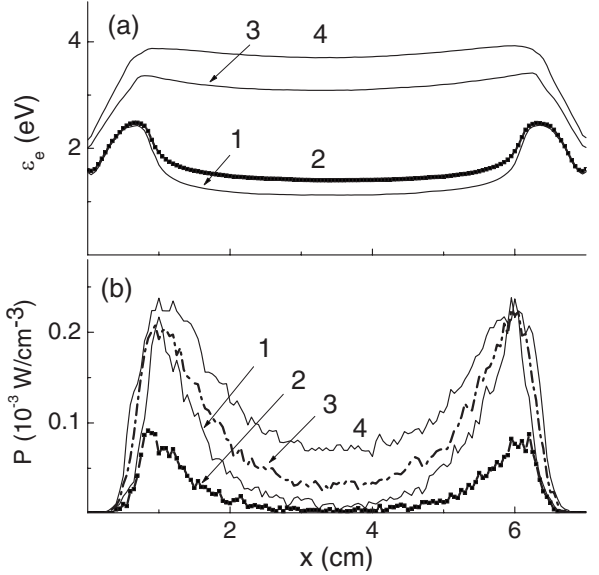

FIG. 3. Distribution of the mean electron energy (a) and discharge power deposited to the ionization (b) for different dust radii: $r_{d}=0$ (1), $20 \mathrm{~nm}(2), 30 \mathrm{~nm}$ (3) and $40 \mathrm{~nm}$ (4).

the midplane [curve 3, 4 in Fig. 3(b)]. Actually the growing dust causes an increase of the electrical field in the plasma, which heats the electrons and enhances the discharge current. The electron energy distribution function (EEFD) in the midplane is shown in Fig. 4 for different nanoparticle radii. It is seen that the EEDFs lose the Maxwellan shape for $r_{d}$ $>30 \mathrm{~nm}$ and the hot electron population becomes larger.

\section{DUST MOTION EFFECT}

In simulations and in experiments, we found that the nanoparticles are accumulated near the sheath-plasma boundaries at the initial growth stage. Note that in previous studies, the dust was considered to be uniformly distributed over the bulk plasma. A void (area without dust) in the plasma was found only for large dust particles. In our case, we observe the void formation for $10-20 \mathrm{~nm}$ nanoparticles and the larger nanoparticles have a more uniform distribution. The measured distribution of the laser light scattering signal from the dust particles and the calculated dust density are shown in Fig. 5. The nanoparticle profile changes the shape from peaked to flat during the C-VD transition, which takes place for $r_{d}>20 \mathrm{~nm}$. In Fig. 6 the mean electron energy in the midplane and the electrode sheath width $l_{s}$ are shown for

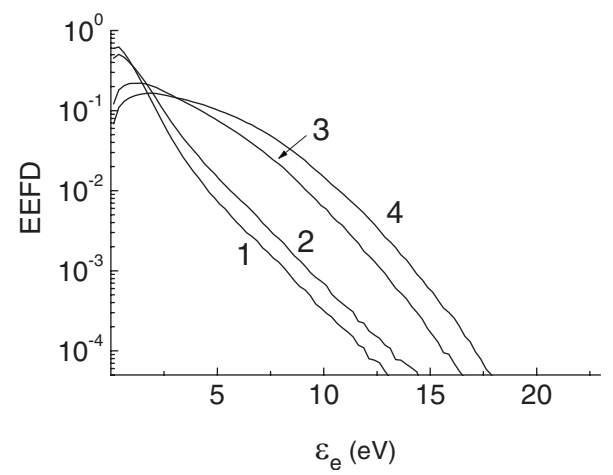

FIG. 4. Electron energy distribution function for different dust radii: $r_{d}=0$ (1), $20 \mathrm{~nm} \mathrm{(2),} 30 \mathrm{~nm} \mathrm{(3)} \mathrm{and} 40 \mathrm{~nm} \mathrm{(4).}$

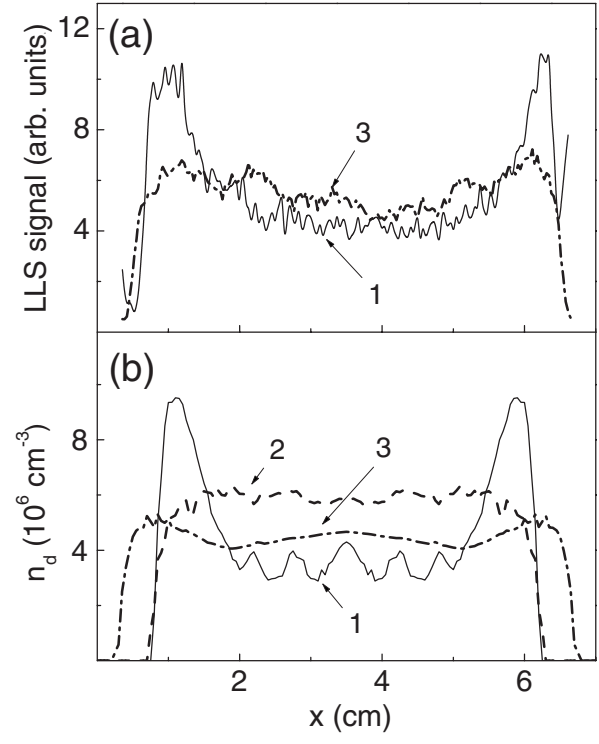

FIG. 5. Distribution of nanoparticles from measured laser light scattering signal (a) and from simulations (b) for different radii: $r_{d}=20 \mathrm{~nm}\left(t=47 \mathrm{~s}\right.$ in the experiment) (1), $r_{d}=30 \mathrm{~nm}(2)$, and $r_{d}$ $=90 \mathrm{~nm}(t=5.8 \mathrm{~min}$ in the experiment $)(3)$.

different dust radii. The $\epsilon_{e}$ and $l_{s}$ remain constant during the first $1 \mathrm{~min}\left(r_{d}<20 \mathrm{~nm}\right)$, while the ionization is large enough to sustain the capacitive mode of discharge operation. For larger $r_{d}$, the $\epsilon_{e}$ increases to provide the ionization in the plasma and this is accompanied with visible sheath compression. As a consequence, the dust surface potential $\phi_{d}$ and dust charge $Z$ increase, because the calculated $\phi_{d}$ and $\epsilon_{e}$ coincide within $20-30 \%$ for our plasma parameters. The larger dust charge requires the larger electrical field to trap it, therefore the sheath width decreases with the dust growth.

Figure 7 shows the ion drag $F_{\mathrm{dr}}$ and electrostatic $F_{\mathrm{el}}$ forces, which increase by a factor of 4 during the C-DV transition. Using the Barnes formula [14], we obtain the ratio of the ion drag to electrostatic force in the bulk plasma (assuming $v_{i} \sim \mu_{0} E$ for subsonic ion motion),

$$
F_{\mathrm{dr}} / F_{\mathrm{el}} \sim Z n_{i} / \epsilon_{i}^{3 / 2}
$$

and near the sheath-plasma boundary (assuming $v_{i} \sim \mu_{1} E^{1 / 2}$ for supersonic ion motion),

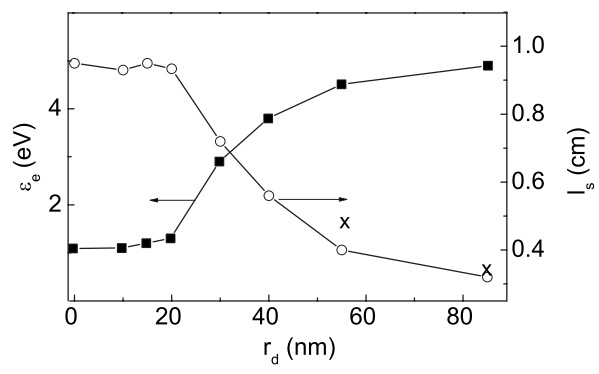

FIG. 6. Calculated sheath width and mean electron energy as a function of nanoparticle radius. Two crosses are from the experiment. 


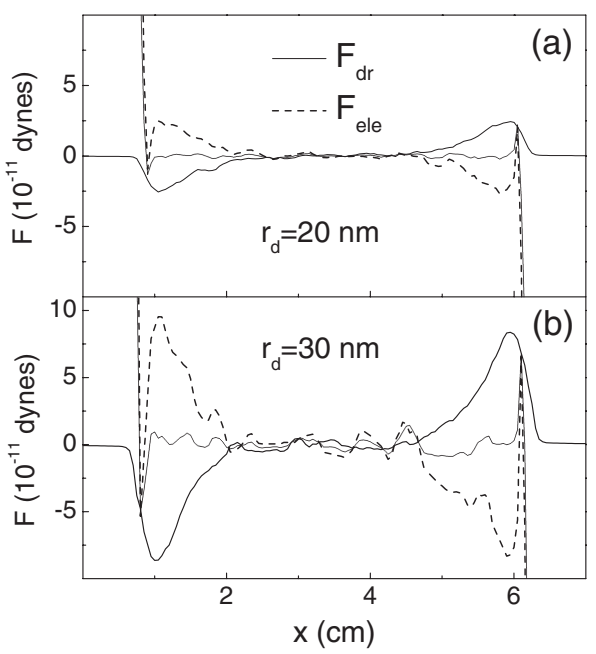

FIG. 7. Computed ion drag (solid lines) and electrostatic force (dashed lines) distributions for $r_{d}=20 \mathrm{~nm}$ (a) and $r_{d}=30 \mathrm{~nm}$ (b).

$$
F_{\mathrm{dr}} / F_{\mathrm{el}} \sim Z n_{i} / E^{1 / 2} \epsilon_{i}^{3 / 2}
$$

In the beginning of the growth, nanoparticles are shifted by the ion drag force to the sheath-plasma boundary. Growing dust induces the C-VD transition, plasma parameters change, and dust distributes uniformly over the plasma. The ion density and electrical field distributions before and after the transition are shown in Fig. 8.

For $30 \mathrm{~nm}$ dust, the ion density is smaller and the electrical field and dust charge are larger, compared to the $20 \mathrm{~nm}$ case. After transition, the ratio $F_{\mathrm{dr}} / F_{\text {el }}$ decreases and dust rearranges uniformly over the plasma. As we discussed in the previous section, the plasma density decreases due to the change of discharge operation regime and an increase of the dust surface area.

The discharge power deposited to the excitation of the background gas by fast electrons also demonstrates the transition between the capacitive and volume-dominated modes. In Fig. 9, the measured light distribution of the ArI $696.5 \mathrm{~nm}$

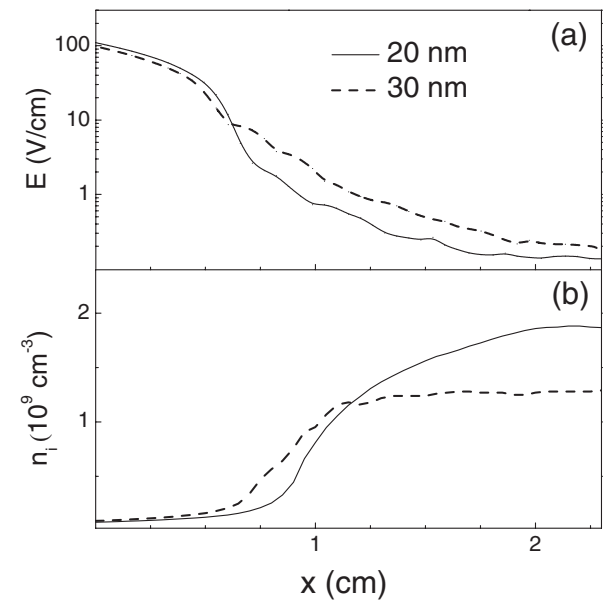

FIG. 8. Electrical field (a) and ion density (b) distributions near the electrode for 20 and $30 \mathrm{~nm}$ dust radii.
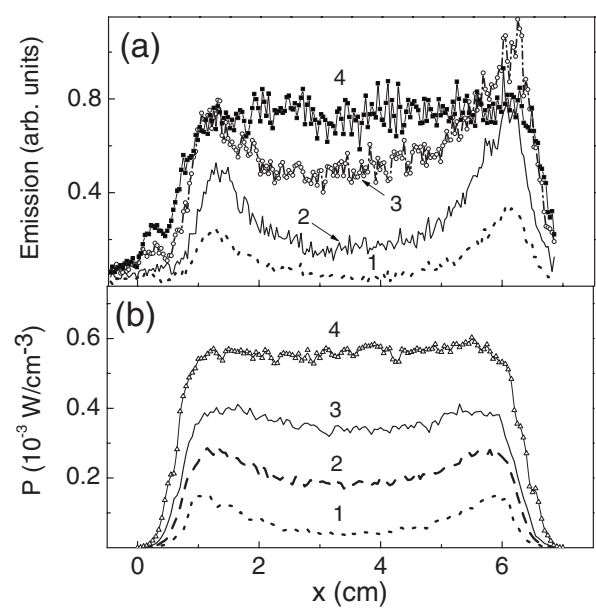

FIG. 9. Light distribution of the ArI $696.5 \mathrm{~nm}$ emission line,

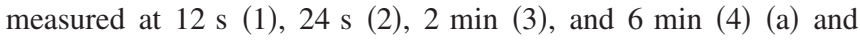
calculated power deposition to excitation of $\mathrm{C}_{2} \mathrm{H}_{2}$ molecules for $r_{d}=20 \mathrm{~nm}$ (1), $30 \mathrm{~nm}(2), 40 \mathrm{~nm}$ (3), and $70 \mathrm{~nm} \mathrm{(4)} \mathrm{(b).}$

emission line and the calculated excitation power are shown. The calculated and experimental profiles are similar and have peaks near the sheath-plasma boundaries for smaller dust radii. This is the characteristic feature of the capacitive mode. For larger dust radii, in the volume-dominated regime the profiles become flat.

We also performed simulations with the unmovable dust with fixed profile given by expressions (6) for the same discharge parameters and dust radii. Under this condition, the transition between different regimes was smoothed and took place at much larger nanoparticle radii. Thus, we found that the consideration of dust motion is very important for an accurate description of transient processes observed in experiments.

\section{CONCLUSION}

In conclusion, we have developed a kinetic model for the CCRF discharge with mobile charged nanoparticles. The dust surface potential, dust motion, and discharge parameters are calculated self-consistently using the PIC-MCC method. In our model, we did not use any assumption about EEDF and IEDF that allows us to calculate accurately the dust particle charging. The results of our simulations and experiments are in good agreement, showing the transient processes induced by the dust presence. Calculations and experiments show the dust accumulation near the sheathplasma boundaries at the initial stage of the nanoparticle growth. At some critical dust size, the transition from the capacitive to volume-dominated regime was observed. At this moment, we also found a quick change of plasma parameters. In the capacitive mode of discharge operation, the ionization generally takes place around the sheath-plasma boundaries, and nanoparticles accumulated here suppress the ionization, absorbing fast electrons. For larger nanoparticles, the ionization is not large enough to compensate the electron and ion losses on the dust surface. Therefore, the discharge transits to more resistive VD mode with the enhanced ion- 
ization in the plasma bulk. The change of plasma parameters initiates the rearrangement of the dust profile from peaked to flat. Further dust growth does not affect the plasma density. The growing absorption of electrons and ions on the dust surface is balanced with an increasing ionization in the discharge plasma. The C-VD transition was monitored with evolution of the plasma density, mean electron energy, ionization rate, and electron energy distribution function. It was shown that the presence of movable dust is responsible for a quick change of plasma parameters observed in the experiments.

\section{ACKNOWLEDGMENTS}

This work was supported by the Bilateral grants RFBRFlanders (Grant No. 05-02-19809-MFa), RFBR-Ukraine (Grant No. 08-02-90446-Uka) and by the Deutsche Forschungsgemeinschaft GK 1051 and SFB 591, project B1 and the Belgian Science Policy (IAP).
[1] E. Kovačević, I. Stefanović, J. Berndt, and J. Winter, J. Appl. Phys. 93, 2924 (2003).

[2] I. Stefanović, E. Kovačević, J. Berndt, and J. Winter, New J. Phys. 5, 39.1-12 (2003).

[3] P. Belenguer, J. P. Blondeau, L. Boufendi, M. Toogood, A. Plain, A. Bouchoule, C. Laure, and J. P. Boeuf, Phys. Rev. A 46, 7923 (1992).

[4] C. Bohm and J. Perrin, J. Phys. D 24, 865 (1991).

[5] J. P. Boeuf and Ph. Belenguer, J. Appl. Phys. 71, 4751 (1992).

[6] I. Denysenko, J. Berndt, E. Kovačević, I. Stefanović, V. Selenin, and J. Winter, Phys. Plasmas 13, 073507 (2006).

[7] M. R. Akdim and W. J. Goedheer, Phys. Rev. E 67, 066407 (2003).

[8] Y. I. Chutov and W. J. Goedheer, IEEE Trans. Plasma Sci. 31,
606 (2003).

[9] R. Lagushenko and J. Maya, J. Appl. Phys. 55, 3293 (1984).

[10] V. V. Ivanov, A. M. Popov, and T. V. Rakchimova, Plasma Phys. Rep. 21, 548 (1995) (in Russian).

[11] M. Hayashi, in Nonequilibrium Processes in Partially Ionized Gases, edited by M. Capitelli and J. N. Bardsley (Plenum, New York, 1990).

[12] C. K. Birdsall and A. B. Langdon, Plasma Physics Via Computer Simulation (McGraw-Hill, New York, 1985).

[13] I. V. Schweigert, V. A. Schweigert, and F. M. Peeters, Phys. Plasmas 12, 113501 (2005).

[14] M. S. Barnes, J. H. Keller, J. C. Forster, J. A. ONeill, and D. K. Coultas, Phys. Rev. Lett. 68, 313 (1992). 